\title{
Building Automation Using IOT With Special Reference Regional Variations
}

\author{
Manuj Darbari \\ Professor \\ Department of Computer \\ Science \\ BBD University \\ Lucknow, India
}

\author{
Ruchi Dave \\ Researcher \\ Department of Computer \\ Science \\ Suresh Gyan Vihar University \\ INDIA, Jaipur,
}

\author{
Naveen Hemrajani \\ Associate Professor \\ Department of Computer \\ Science \\ JECRC University \\ INDIA, Jaipur,
}

\begin{abstract}
This paper discusses the Mood based ambient settings using the concept of BACM (Building Automation and Cultural support Model). In order to model the system we have used GAIA methodology for Multiagent Sensor network which is integrated with BACnet for Agent synchronization. Finally the model is verified using Process Algebra.
\end{abstract}

\section{General Terms}

Intelligent Control System

\section{Keywords}

Home Automation system, BACM, Process Algebra.

\section{INTRODUCTION}

Our research deals with combination of interdisciplinary fields such as building automation by looking into various aspects of cultural variations. When we start integrating these two objectives we have to look into various artifacts and environments which deals with capturing various styles of living these architectural viewpoints and particular considerations of material types. There are variety of challenges which are linked to building automation according to cultural diversity. Our main aim is to generate maximum ideas about different modeling techniques for building automation. We choose a Method Evaluation Model (MEM) (moody 2003) to develop our framework [1,2,5,6,8,9]



Figure 1: MEM model (Moody 2003)

We have to model the building automation based on cultural information of that area using Technology Acceptance Model (TAM) (David, 1989) based on two basic criteria's:

- The Effectiveness and Usefulness of the system The derived benefit which the user will find relating to usability aspect of the system depends on performance and the behavior of a method using qualitative and quantitative methods.

This qualitative model consist of simple Agent model and its quantitative model. The methodology used for Agent model includes MaSE. Multiagent Software Engineering which is also the basis for agent Tool development system.

\section{CURRENT SYSTEM ON BUILDING AUTOMATION}

There is tremendous work done in the area of building automation ranging from normal one to one control of the devices to the use of web services as the mode to control. . the article by Sarah Erdman , on "See Your Building - Anywhere ". anytime successfully develop a IP based system to put the entire home under surveillance. Secondly "Prediction for Smart Building in 2011." By Jim Sinopoli and Mashup and Billal's paper on "Is wireless technology ready for the arena?" highlighted the concept of how wireless technologies can integrate various home appliances. Lastly "Total Facility Control" by Ron Bernstein and "Custom Automation" highlights smart building and lighting control.

\section{MODEL DEVELOPMENT}

The main aim of the model development is the ease of use. The perceived usefulness and to develop a formal rule based BACM (Building Automation and Cultural support Model) and finally convert it into some formal quantitative model.

As move across length and breadth of India we come across various cultural diversity which occurs because of its regional variations and climatic conditions. If we are designing a house located in Himanchal Pradesh then we have to support their basic needs like heating system and their style of living while we are designing for South India other parts then we need to focus on cooling system.

In order to organize full automation of the system we use the concept of BACnet, it refers to Data Communication Protocol for Building Automation and Control Networks. It works as an independent an independent, open source protocol for control and automation. It can be implemented directly on top of IEEE 802.2 data link layers. The unique thing about BACnet is it follows the OSI model but doesn't have Transport and Session layers. BACnet assumes each device as objects. Each object is identified by its unique Object_Identifier within the device. BACnet provides excellent alarm and event services, messages are sent ANSI system. It supports multiple events reporting options using "Intrinsic Reporting" like status updates etc.

It starts with (figure 1) knowledge base creation[3,4,7] using sensors which interacts with the existing knowledgebase which acts as cognitive database generator similar to FIS controller. 


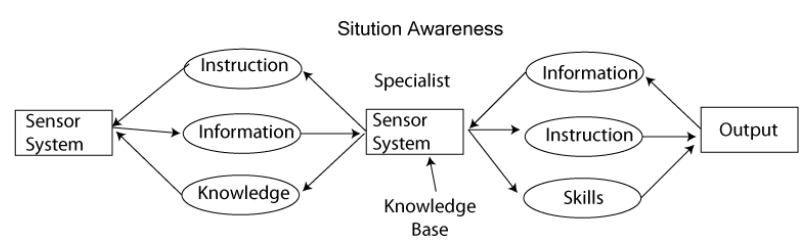

Figure 2: Situation awareness diagram

To provide an integrated environment we use the concept of Multi-Agent. This helps us in coordinating various devices with control circuit and each device can also considered as an intelligent $[10,12,14]$ and self reliant entity. The basic advantage of using Multiagent system is Agent's ability to coordinate with each other.

The Multi-Agent system[11,13] which we used is GAIA methodology. The main concept of GAIA is divided into two broad categories: Abstract and Concrete.

Abstract entries are those used during analysis to visualize the system but it is not necessarily being realized within the system.

Concrete entities are those used during analysis to visualize the system but it is not necessarily being realized within the system.

Concrete entities are used within the process of design and have direct correspondence in the run time system.

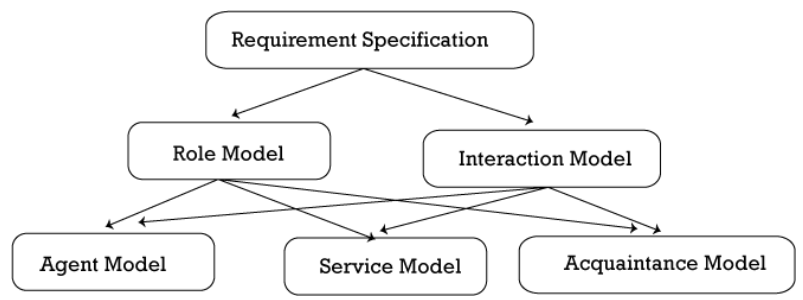

Figure 3: GAIA methodology Framework

Table 3-1: Role Modeling using GAIA

\begin{tabular}{|c|l|}
\hline Operator & Interpretation \\
\hline$x \cdot y$ & $\mathrm{x}$ followed by $\mathrm{y}$ \\
\hline$x \mid y$ & $\mathrm{x}$ or y occurs \\
\hline$x^{*}$ & $\mathrm{x}$ occurs 0 or more times \\
\hline$x$ & x occurs 0 or more times \\
\hline$x^{\omega}$ & $\mathrm{x}$ occurs infinitely often \\
\hline$[x]$ & $\mathrm{x}$ is optional \\
\hline$x \| y$ & $\mathrm{x}$ and y are interleaved \\
\hline
\end{tabular}

For instance Control Agent is responsible for reciving the signals and controlling the fixtures accordingly can be represented as:

$$
\begin{aligned}
\text { Control Agent }+ \text { Provider Agent } \\
=\text { Home Automation Agent }
\end{aligned}
$$

For collaboration amongst sensor we have used the work by Thomos W. Malore which focuses on coordination of components where each entity is considered as object and functionality its activities.
Highlighting the problem further we use recommender system based on the facial expression of the person depending on the eyes and check images which are pre-stored a recommender system will run which will initiate a particular set of mood setting of sensors. It consists of Facial Record, Knowledge base, generic sensor setting and knowledge model.

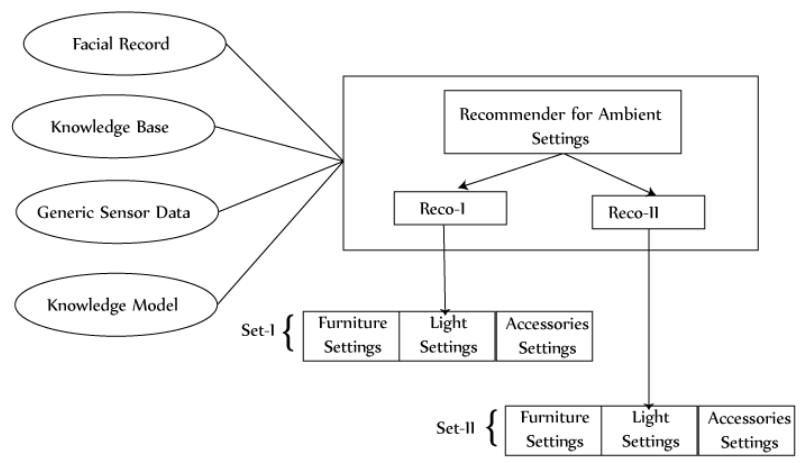

Figure 4: Recommender System

Now defining the Role Modeling Using GAIA we have:

Table 3-2: Role Modeling using GAIA

ROLE: Control Agent

Description: Attending signals from Camera, receive Recommendation from Knowledge Base, Select best combination, Query Schedule Information.

PROTOCOLS and ACTIVITIES: Receive and Request signal data, Send schedule request setting and receive response (Camera Response).

PERMISSION: Select alter prerecorded combinations.

\section{RESPONSIBILITIES:}

Control Agent $=$

Control Agent $=$

Control Agent $=$
(Sensors, Camera, Devices) (Recommendations) ${ }^{\omega}$

(Transfer of New Setting to Record Agent) ${ }^{\omega}$
Extending further the GAIA agents Mobile devices[15] are programmed according to GAIA entities and IoT cloud framework (figure 2) known as Mood based environment Adjustment. Table 3-2 and Figure 4 shows the intelligent agent coordination achieved through GAIA as the camera read the mood of the person sitting on the sofa and the facial expressions (figure 5) are read by the camera and based on that the reclining of sofa, setting of a particular channel of TV and sending message on users mobile about the items in the refrigerator which can be cooked instantaneously (figure. 7 and 8). 


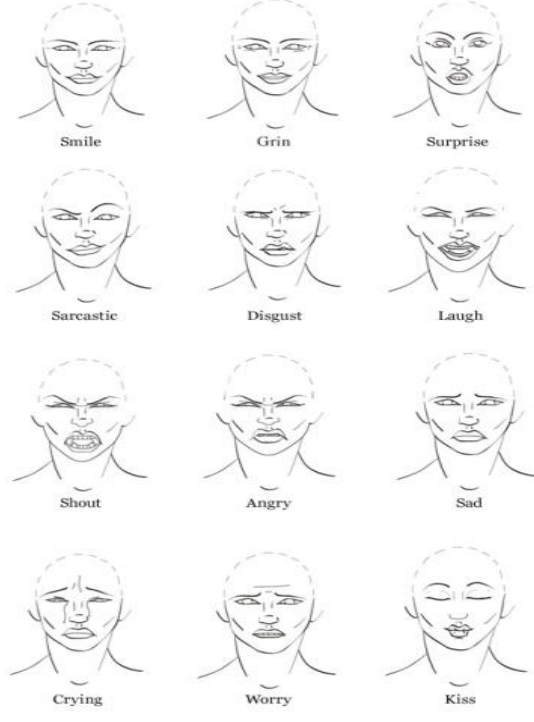

Figure 5: Various types of facial expressions

Based on the facial expression of the user, MOOD system identifies the correct MOOD action/effect process algebra (fig. 5)

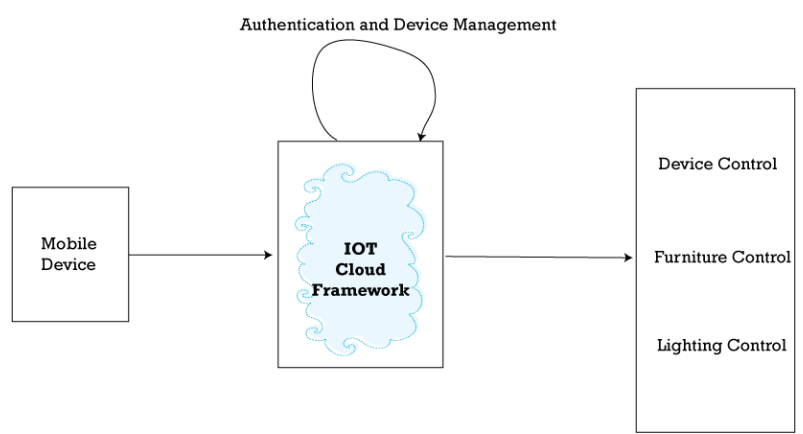

Figure 6: IoT cloud framework for device and sensor management



Figure 7: Mood based environment adjustment

On the other side user can also give command through his mobile phone (SMART HOME CONTROL Software) to control the ambient setting including mood based lighting system. We have used Arduino board and sensor network as a part of IoT framework.
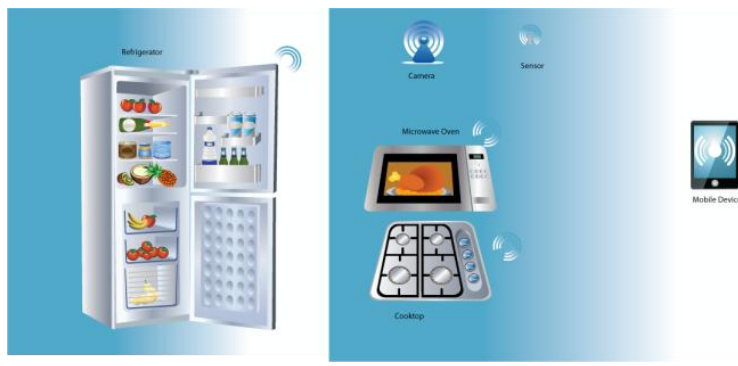

Figure 8: Smart Appliances

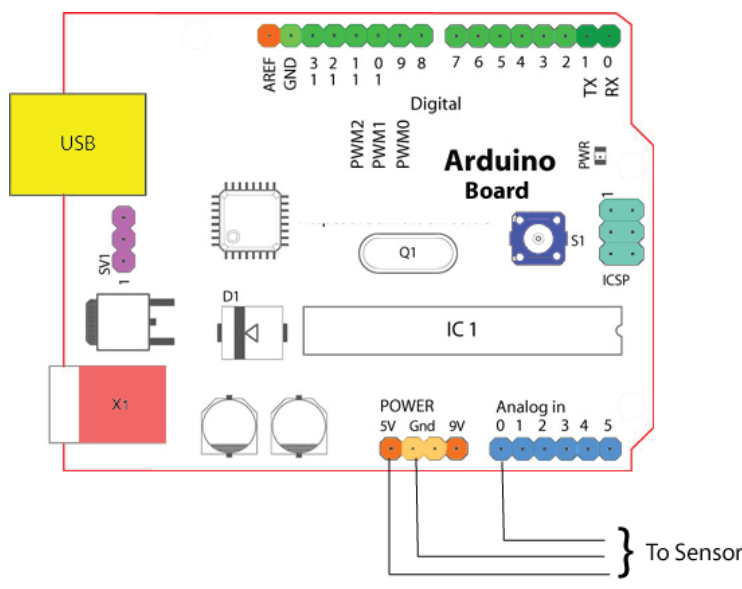

Figure 9: Arduino board

The entire set of activities can be formalized by sequence flow diagrams showing how agents participate collaboratively in the system.

To apply a coherency between various sensor types we establish mathematical foundation using process algebra. The reason of using Process Algebra is a parallel operator which is done to break down the system into concurrent components in order to test non-trivial properties of the system. It is very well suited for mission control operation. Each atomic action ' $a$ ' is a constant that can execute itself, after which it terminates successfully:

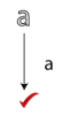

The predicate $\stackrel{a}{\longrightarrow} \checkmark$ represents successful termination after the execution of action ' $a$ '.

Parallel operations are defined as ' +' and product ' '. in case if the person wants the agent to perform the operations or camera sensors gestures to perform some operation automatically, meaning that two processes are to executes simultaneously. Let ' $u$ ' be user defined process and ' $m$ ' mood based setting depending on facial gestures. $u \| m$ can choose to execute an initial transition of $u$ (i.e. $u \stackrel{a}{\rightarrow} u^{\prime}$ or $u \stackrel{a}{\rightarrow} \square$ ) an initial transition of $t$. We can choose to execute a communication between initial transition of $u$ and $m$.

In order $u$ and $m$ to communicate a set of clusters of operation $a$ and $b$ are executed and $c$ be defined the final state of $u$ and $m$ to be reached in order to complete as per specification.

Let $c=$ comfort level; which is supposed to be set at highest level no matter what is the combination of $a$ and $b$.

Let $a=H V A C \&$ Ambient Lighting $(H A)$ 


\section{$b=$ Peripheral Device Combination (PDC)}

In any combination of $H A$ and $P D C$ we can draw a process graph of sensor settings but all the combination should arrive at providing highest level of comfort $c$.

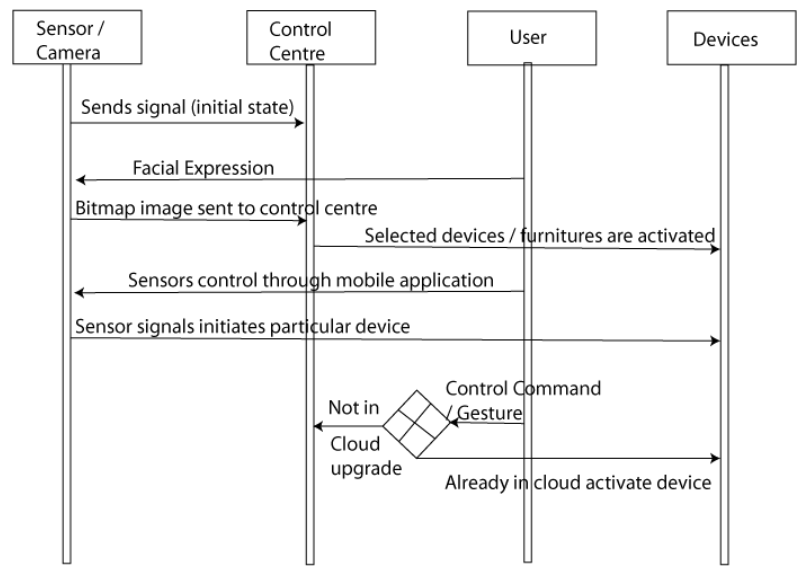

Figure: Process graph of sensors

The above figure shows that the merge of two simple process items produces a relatively large process graph. The use of process algebra defines the state in the protocol showing the transitions between different states.

Let $E$ consists of linear recursive equations given as:

$$
X_{i}=a_{i 1} X_{i 1}+\cdots+a_{i k} X_{i k}+b_{i 1}+\cdots+b_{i l_{i}}
$$

where $i$ ranges from $\{1,2, \ldots, n\}$

In order to specify that the entity setup of sensor net we have correlate action and effect. If the camera experiencing hard mood then reclining of chair and ambient lighting as well as Air Conditioner/ Heating system should be adjusted at LevelII.

If very light mood then ambient can be formulated using the following rules:

Let $i$ define the range from $L O W$ to $H I G H$.

$j$ define the range from $H I G H$ to LOW.

Thus $\lambda_{s}(t)$ can execute the visible behavior action $(s, a)$ of an initial $a$-transition of $t$ in state $s$; the action $(s, a)$ is a successful termination of the transition.

According to Process Algebra we can write the equation for the coordination as:

$$
\begin{aligned}
\lambda_{s}(t)=\operatorname{action}(s, & \left.a_{i 1}\right) \lambda_{\text {effect }}\left(s, a_{i 1}\right)+\cdots \\
& +\operatorname{action}\left(s, a_{j 1}\right) \lambda_{\text {effect }}\left(s, a_{i j}\right)+\cdots \\
& +\operatorname{action}\left(s, b_{i m}\right)
\end{aligned}
$$

The above equation represents the mood for the first instance typesetting similarly we can develop equations for other mood combinations.

In order to model the above situation we have used "Data parameters" for passing value or the real time values from Ambient. The data parameters are passed using pre and post conditions which mean the mood is happy under particular conditions Ambient needs to be set at III level and so on

\section{CONCLUSION}

Our work focuses on integration perspective of various sensor networks with special emphasis on mood based control system design. We have used ZigBee based automation system design since its low power consumption and ease of integration. For capturing the images we have used various forms of facial expression based on which reclining of sofa and ambient lighting system will change, besides that user can also change his surroundings from his mobile.

In future we are trying to automate using the concept of Multiobjective Optimisation where the entire setting can be optimized to its best level.

\section{REFERENCES}

[1] Bansal, S., \& Darbari, M. (2012). Application of multi objective optimization in Prioritizing and Machine scheduling: a Mobile Scheduler Toolkit. International Journal of Advance Information Systems, 3(2).

[2] Cernuzzi, L., Molesini, A., Omicini, A., \& Zambonelli, F. (2011). Adaptable multi-agent systems: the case of the gaia methodology. International Journal of Software Engineering and Knowledge Engineering, 21(04), 491521.

[3] Darbari, M., Asthana, R., \& Singh, V. K. (2010). Integrating Fuzzy Mde-AT Framework for urban traffic simulation. International Journal of Software Engineering, 1(1).

[4] Darbari, M., Asthana, R., Ahmed, H., \& Ahuja, N. J. (2011). Enhancing the capability of N-dimension selforganizing Petrinet using neuro-genetic approach. International Journal of Computer Science Issues (IJCSI), 8(3).

[5] Darbari, M., Singh, V. K., \& Kumar, G. (2014). Coalescence of Evolutionary Multi-Objective Decision Making approach and Genetic Programming for Selection of Software Quality Parameter. Complexity, $7(11)$.

[6] Darbari, M., Srivastava, A. K., \& Medhavi, S. (2009). PENTRAL: pattern based logic language. Journal of International Technology and Information Management, 18(3-4), 385.

[7] Dhanda, N., Darbari, M., \& Ahuja, N. J. (2012). Development of Multi Agent Activity Theory eLearning(MATeL) Framework focusing on Indian Scenario. International Review on Computers and Software, 7(4).

[8] Fokkink, W. (2013). Introduction to process algebra. Springer Science \& Business Media.

[9] Lavania, S., Darbari, M., Ahuja, N. J., \& Siddqui, I. A. (2015). Genetic Algorithms-Fuzzy Based Trade-Off Adjustment between Software Complexity and Deliverability. In New Trends in Networking, Computing, E-learning, Systems Sciences, and Engineering (pp. 15-18). Springer International Publishing.

[10] Singh, P., Kapoor, A., Kaushik, V., \& Maringanti, H. B. (2012). Architecture for automated tagging and clustering of song files according to mood. arXiv preprint arXiv: 1206.2484 .

[11] Tahilyani, S., \& Darbari, M. (2015). Cognitive Framework for Intelligent Traffic Routing in a Multiagent Environment. In Software Engineering in Intelligent Systems (pp. 67-76). Springer International Publishing. 
[12] Tahilyani, S., Darbari, M., \& Shukla, P. K. (2012). A new multi agent cognitive network model for lane-bypass approach in urban traffic control system. International Review on Computers and Software, 7(5).

[13] Wooldridge, M., Jennings, N. R., \& Kinny, D. (2000). The Gaia methodology for agent-oriented analysis and design. Autonomous Agents and multi-agent systems, 3(3), 285-312.
[14] Yagyasen, D., Darbari, M., Shukla, P. K., \& Singh, V. K. (2013). Diversity and convergence issues in evolutionary Multiobjective optimization: application to agriculture science. IERI Procedia, 5, 81-86.

[15] Zambonelli, F., Jennings, N. R., \& Wooldridge, M. (2003). Developing Multiagent systems: The Gaia methodology. ACM Transactions on Software Engineering and Methodology (TOSEM), 12(3), 317370 